\title{
Transverse compression of tendons
}

\author{
S. T. Samuel Salisbury \\ Department of Engineering Science, University of Oxford, \\ Parks Road, Oxford OX1 3PJ, UK \\ sam.salisbury@oxon.org \\ C. Paul Buckley \\ Department of Engineering Science, University of Oxford, \\ Parks Road, Oxford OX1 3PJ, UK \\ paul.buckley@eng.ox.ac.uk
}

\section{Amy B. Zavatsky*}

Department of Engineering Science, University of Oxford, Parks Road, Oxford OX1 3PJ, UK

amy.zavatsky@eng.ox.ac.uk

Accepted for publication on 11 January 2016

\footnotetext{
* Corresponding author.
} 
Journal of Biomechanical Engineering

\section{ABSTRACT}

A study was made of the deformation of tendons when compressed transverse to the fiber-aligned axis. Bovine digital extensor tendons were compression tested between flat rigid plates. The methods included: in situ image-based measurement of tendon crosssectional shapes, after pre-conditioning but immediately prior to testing; multiple constant-load creep/recovery tests applied to each tendon at increasing loads; and measurements of the resulting tendon displacements in both transverse directions. In these tests, friction resisted axial stretch of the tendon during compression, giving approximately plane strain conditions. This, together with the assumption of a form of anisotropic hyperelastic constitutive model proposed previously for tendon, justified modeling the isochronal response of tendon as that of an isotropic, slightly compressible, neo-Hookean solid. Inverse analysis, using finite element simulations of the experiments and $10 \mathrm{~s}$ isochronal creep displacement data, gave values for Young's modulus and Poisson's ratio of this solid of $0.31 \mathrm{MPa}$ and 0.49 , respectively, for an idealized tendon shape and averaged data for all tendons; and $E=0.14$ and $0.10 \mathrm{MPa}$ for two specific tendons using their actual measured geometry. The compression load versus displacement curves, as measured and as simulated, showed varying degrees of stiffening with increasing load. This can be attributed mostly to geometrical changes in tendon cross-section under load, varying according to the initial 3D shape of the tendon.

Keywords: tendon, compression, neo-Hookean model, finite element model, Young's modulus, Poisson's ratio 
Journal of Biomechanical Engineering

\section{INTRODUCTION}

Tendons in vivo experience primarily tensile loading along their fiber-aligned axes. But they also undergo compression and shear. Some of these more complex loadings occur normally, as when a tendon wraps around a bone and is subjected to longitudinal tension and transverse compression. Other complex loadings are abnormal and may lead to tendinopathy [1] or tendon rupture. Most previous mechanical testing of tendons has been performed in tension along their fiber-aligned axes. No unambiguous, quantitative study of tendon deformation under transverse compression has yet been reported. The present work aims to provide this. Such information is needed for constructing three-dimensional (3D) constitutive models of tendon, required for 3D biomechanics simulations in which tendons are involved.

The testing of tendons under an applied load in the transverse plane is challenging, in view of their small transverse dimensions. Nevertheless two studies have achieved measurements on specimens with all dimensions as small as the tendon width, cut out from whole tendons. Lynch et al. [2] tested dumb-bell-shaped specimens cut from sheep flexor tendons, in both longitudinal tension and transverse tension, and found the longitudinal toe-region and linear-region Young's moduli to be two orders of magnitude greater than the transverse moduli. They also found the average Poisson's ratio values to be six times larger for loading in the longitudinal direction than for loading in the transverse direction. Williams et al. [3] loaded small samples cut from rabbit patellar tendon in compression in both longitudinal and transverse directions $(6 \mathrm{~mm}$ diameter disk specimens were used for the latter). In the 'small strain' region, they found the apparent 
Journal of Biomechanical Engineering

Young's modulus in compression to be about one order of magnitude lower in the fiberaligned direction than in the transverse direction.

Some authors have subjected tendons to localized transverse compression in indentation tests. Lee et al. [4] used this approach to study inhomogeneity in the human supraspinatus tendon. Ikeda et al. [5] and Dourte et al. [6] used it to quantify the effects of different chemical treatments on the transverse compressive properties of canine and mouse tendons. However, indentation tests involve complex inhomogeneous deformations, so quantitative measurements of macroscopic elastic constants could not be obtained.

Main et al. [7] overcame the intrinsic problems of the above methods by measuring the transverse compression of whole tendons. They applied an axial pre-load to digital flexor tendons from the human forearm, and then compressed them quasistatically in the transverse direction between convex cylindrical platens of diameter 10 $\mathrm{mm}$. The axes of the curved platens were aligned perpendicular to the tendon axis. Main et al. then employed inverse analysis, comparing measurements of force versus compression with finite element (FE) predictions, to determine apparent values of two elastic constants of the tendon. The tendons were approximated as incompressible cylinders with elliptical cross-section. A single-term Ogden hyperelastic constitutive model was assumed: the two constants obtained were the two parameters of the singleterm Ogden model. From the average value of one of the constants, an apparent shear modulus of $18 \mathrm{kPa}$ may be deduced. However, the literal meaning of this value is uncertain. The experimental configuration allowed three-dimensional deformation throughout the tendon, with axial expansion in addition to transverse compression. Real 
Journal of Biomechanical Engineering

tendons are strongly anisotropic and would show negligible axial expansion under these conditions, whereas the simulated tendon represented by the isotropic Ogden model would expand significantly.

The present study follows an approach similar to that of Main et al in testing whole tendons and using inverse analysis to determine tendon properties, but is designed to improve accuracy in modeling the strain field. Whole tendons were subject to transverse compression over lengths much greater than their thickness: typically $\mathrm{x} 10$. Under these conditions, friction constrains the axial displacement, producing approximately plane strain conditions, irrespective of the degree of material anisotropy. This is the method exploited successfully by Ward and co-workers [8-10] and others, for studying the elastic anisotropy of anisotropic (but transversely isotropic) synthetic polymer fibers. It consists of transversely compressing a cylindrical specimen, with length:diameter $>>1$, between flat, rigid plates, and simultaneously measuring the load and at least one of (a) the width of the contact patch [8], (b) the diametral expansion perpendicular to the load [9], or (c) the diametral contraction parallel to the load [10]. For the special case of a linear elastic, transversely isotropic cylinder of circular crosssection, subject to infinitesimal strains, the full two-dimensional stress and strain fields can be solved analytically, as shown by the above authors [8-10]. In addition to those, several other studies have exploited the method to determine transverse elastic constants of plant fibers [11], textile fibers [12], and high performance reinforcing fibers [13,14].

Application of the method to tendons exploits the fact that they are approximately cylindrical in shape. Of course, the cross-section is not usually circular, and finite deformations are of practical interest, so the analytical solutions referred to above do not 
Journal of Biomechanical Engineering

apply. But this limitation is overcome here, by employing quantitative determination of the actual tendon cross-sectional geometry, followed by numerical simulations of the experiment combined with inverse analysis of the data.

\section{METHODS}

\subsection{Experimental set-up}

Bovine digital extensor tendons were tested in compression transverse to the fiber-aligned direction using a specially designed rig (Fig. 1), which also allowed measurement of tendon cross-sectional shape and area [15] (Fig. 2a).

Each tendon sample was hung vertically (in the $Z$ direction) under its own weight and during testing was compressed horizontally (in the $Y$ direction) at mid-substance between two flat glass plates. One (the glass backing) was fixed rigidly to an aluminum backing plate. The other was an optically flat glass plate (Comar Instruments 63 GQ 40, Cambridge, UK) fitted over a window in the rigid paddle used to compress the tendon. Paddle position in the $Y$ direction was measured with an LVDT displacement transducer (RDP Electronics DCT-500A, Wolverhampton, UK). A digital video camera (Basler A631f, Ahrensburg, Germany) recorded a side view of the specimen (in the XZ-plane, see Fig. 1) through the glass plate.

The MATLAB (The MathWorks, Natick, Massachusetts, USA) imageacquisition, data-acquisition, and image-analysis toolboxes were used for data acquisition and processing. An aluminum calibration grid [15] was imaged and used to find a pixelto-mm conversion factor. From the intensity profiles of the digital images, the initial and 
current visible widths, $\Delta X_{0}$ and $\Delta X$, of the tendon were measured, and hence the $X$-wise change (expansion) in width of the tendon was determined $\delta_{x}=\Delta X-\Delta X_{0}$. The $Y$ displacement of the tendon was taken to be the $Y$-displacement of the center of the glass plate, calculated using the paddle position measured by the LVDT and the geometry of the loading mechanism (Fig. 1a) ${ }^{1}$. Hence changes in the separation $\Delta Y$ of the centers of the glass plates gave the $Y$-wise change (contraction) in thickness of the tendon in the loading direction $\delta_{y}=\Delta Y_{0}-\Delta Y$. The compressive load applied to the tendon at the center of the glass plate was calculated by balancing the moments about the pivot of the loads on the paddle (Fig. 1a).

\subsection{Experimental protocol}

Ten digital extensor tendons, each approximately $160 \mathrm{~mm}$ in length, were dissected from bovine feet immediately after slaughter, and quickly moved to a freezer at $-18^{\circ} \mathrm{C}$ for storage, to minimize any drying [16]. Tendons were returned to room temperature the day before testing and soaked in $10 \%$ sucrose solution overnight and until the time of testing. The purpose of this step was to achieve a degree of hydration as close as possible to that of a freshly harvested tendon [17]. Immediately prior to testing, tendon surfaces were rinsed with phosphate-buffered saline (PBS) to remove any stickiness arising from the sucrose. All tests were carried out at room temperature $c a$ $20^{\circ} \mathrm{C}$.

\footnotetext{
${ }^{1}$ The finite width of the tendon meant that compressive displacement varied by $\pm 3 \%$ from the mean across the width of the tendon. However, this was small in comparison to the variation of displacement caused by unevenness in thickness of the tendon across its width and length: typically ca 10\% - see Figure 2.
}

BIO-15-1191 
The protocol applied to each of seven tendons was as follows. One end of the tendon was mounted vertically in the drill chuck serving as upper sample clamp, and allowed to hang freely under its own weight ${ }^{2}$. The tendon was pre-conditioned by application of ten $20.8 \mathrm{~N}$ compressive $10 \mathrm{~s}$ on / $10 \mathrm{~s}$ off cycles of transverse load. Then the cross-sectional shape and area were measured at five locations along the tendon, from $1 \mathrm{~mm}$ below to $1 \mathrm{~mm}$ above the glass window, following the method described previously [15]. Afterwards, the tendon was immediately immersed in a $10 \%$ sucrose solution bath for 1 hour under no load, while it was still mounted in the test rig.

Following this soak, the tendon was subject to ten compressive creep/recovery load profiles with the magnitude of the load increasing from $0.9 \mathrm{~N}$ to $39.7 \mathrm{~N}$. Each profile was of duration $c a 15 \mathrm{~min}$, with a one-hour recovery period under no load in the $10 \%$ sucrose solution between each, followed by a surface rinse with PBS. The profile consisted of: (1) application of a pre-load of $0.9 \mathrm{~N}$ for $5 \mathrm{~s}$, followed by its removal and 50 s recovery (sufficient for full recovery of all recoverable strain from this load); and then (2) application of the main creep load for $120 \mathrm{~s}$, followed by its removal and $660 \mathrm{~s}$ of strain recovery. Each application or removal of load took up to $c a 1 \mathrm{~s}$. The peak values of $X$ and $Y$ displacements reached during the $5 \mathrm{~s}, 0.9 \mathrm{~N}$, pre-load were taken as the zero points for $X$ - and $Y$-displacements, respectively. The main creep loads were chosen on the basis of results from preliminary tests, to ensure that the highest load was sufficiently large to provide significant deformation of the tendon sample, but not large enough to permanently damage the tissue.

\footnotetext{
${ }^{2}$ From measurements of the toe-region axial tensile modulus of tendons from the same batch [19], the axial tensile strain produced by the self-weight of the tendon can be estimated as less than $10^{-5}$. This was neglected.
} 
Journal of Biomechanical Engineering

The other three tendon samples were used to quantify repeatability of the experiments. Three compressive loads $(2.8 \mathrm{~N}, 20.8 \mathrm{~N}, 39.7 \mathrm{~N})$ were applied twice to each tendon using the same procedure as for the previous seven tendons, but excluding the cross-sectional area measurements. From this data, repeatability of the $Y$-displacement, for repeat tests on the same tendon across the range of loads applied, was calculated (at the $95 \%$ confidence level) using the method advocated by Hayward [18] and found to be $\pm 0.04 \mathrm{~mm}, 10 \mathrm{~s}$ after load application [19].

The contact condition between the glass plates and a tendon sample was characterized as follows. A tendon was mounted in the compression rig but not clamped at its top end. Instead, this end was placed just below the top of the glass window. A transverse compressive load of $9.45 \mathrm{~N}$ was applied to the tendon, and an increasing longitudinal tensile load was then applied to the bottom of the tendon. Apparent solidsolid friction was observed: the tendon remained stationary until a critical tensile load ${ }^{3}$ was applied, when slipping commenced. This test was repeated five times, and the apparent coefficient of static friction $\mu_{\mathrm{s}}$ was found to be $0.35 \pm 0.01$. In numerical simulations of the tests, Coulomb friction was assumed, with this value of $\mu_{\mathrm{s}}$.

\subsection{Finite element simulations}

Finite element simulations of the transverse compression test were used in the processing of experimental force-displacement data, to deduce best-fit values of the elastic constants. Two approaches were used, to allow their performances to be

\footnotetext{
${ }^{3}$ The contribution to this load arising from the self-weight of the tendon was neglected. The resulting error in $\mu_{\mathrm{s}}$ was less than the scatter in the measurements.
}

BIO-15-1191

Zavatsky 
Journal of Biomechanical Engineering

compared. They employed two different routes for the creation of 3D solid models for the finite element analysis.

The first approach, for a given tendon, was to use its five actual measured crosssections, to create a tendon-specific 3D solid model. In view of the irregularity of crosssectional shape (Fig. 2), this approach required a relatively high mesh density to be used. Consequently, the iterative inverse analysis was then relatively expensive in CPU time see below.

The second approach, which used an idealized tendon model, was more economical in terms of mesh density and CPU time required. It was also more robust, avoiding convergence problems found with some of the tendon-specific models (see below). It produced values for the elastic constants representative of the whole set of seven tendons. From the full set of 21 central cross-sectional profiles (Fig. 2a), a 'representative' cross-sectional profile (Fig. 2b) was calculated using the imageprocessing toolbox in MATLAB and following the procedure described in Salisbury et al. [15]. An analytical 'race-track' shape (two flat sides connected by semi-circular ends, Fig. 2b) was then fitted to this 'representative' cross-section by taking the $Y$-direction thickness of the race-track to match the height of the representative profile $(4 \mathrm{~mm})$. The $X$-direction width of the racetrack was then taken to match the width of the representative profile $(10 \mathrm{~mm})$. This race-track cross-section was extruded along the whole length of the tendon, to create the idealized tendon solid model.

In each approach, cross-sectional profiles were imported into a finite element preprocessor (Patran v.2005.0701, MSC Software, California, USA) and used to create lofted solid models, which were then imported into the finite element software package 
(ABAQUS CAE, v. 6.6-1, Rhode Island, USA). The top and bottom cross-sectional profiles were extruded axially to represent the regions of the tendon at its ends, not in contact with the glass plates of the testing rig. Each tendon solid model was partitioned into three sections: one in the mid-section (contacting the glass plates) and one at each end (not in contact with the glass plates). Automatic meshing of each of the partitions was performed in ABAQUS CAE using 10-node quadratic tetrahedron elements (ABAQUS C3D10M [20]). The mesh density was chosen on the basis of results of 14 preliminary analyses, investigating the effects of varying mesh density on predicted loads, in order to ensure well-converged solutions. The meshes finally used contained $c a$ 13000 elements and 30000 nodes for the tendon-specific models, and 3299 elements and 5749 nodes for the idealized tendon model. In this way, 3D tendon models of two types were created for analysis: models with tendon-specific geometries and one with the idealized tendon geometry.

The inverse analysis approach requires the a priori assumption of a constitutive model, whose form is known to be a good representation of the material. However, there is currently insufficient knowledge of the anisotropic nonlinear viscoelasticity of tendon to justify using a particular representation of it in the inverse analysis. Therefore, in the present finite element simulations, the role of time was not considered. An elastic material model was employed, and displacements in the FE simulations were compared with measured isochronal creep displacements for a particular creep time. See the Appendix for explanation of the justification for this approach. Thus tendon tissue was approximated as a transversely isotropic, slightly compressible, hyperelastic solid. The Cauchy stress is then fully defined by a strain energy density function $W$, depending on 
five independent invariants of the right Cauchy Green tensor $\boldsymbol{C} \equiv \boldsymbol{F}^{\mathrm{T}} \boldsymbol{F}$ (where $\boldsymbol{F}$ is the deformation gradient) and a structural tensor $\boldsymbol{M}_{0} \equiv \boldsymbol{a}_{0} \otimes \boldsymbol{a}_{0}$, formed from unit vector $\boldsymbol{a}_{0}$ specifying the initial direction of the unique axis of the material - see Spencer [21] and Holzapfel [22]. Thus, $W=W\left(I_{1}, I_{2}, I_{3}, I_{4}, I_{5}\right)$, where

$$
I_{1} \equiv \operatorname{tr} \boldsymbol{C}, I_{2} \equiv \frac{1}{2}\left[(\operatorname{tr} \boldsymbol{C})^{2}-\operatorname{tr} \boldsymbol{C}^{2}\right], I_{3} \equiv \operatorname{det} \boldsymbol{C}, I_{4} \equiv \operatorname{tr}\left(\boldsymbol{C} \boldsymbol{M}_{0}\right), I_{5} \equiv \operatorname{tr}\left(\boldsymbol{C}^{2} \boldsymbol{M}_{0}\right)
$$

In the present work, a slightly restricted form for $W$ was assumed, invoking two assumptions: (1) the dependencies of $W$ on $I_{1}, I_{2}, I_{3}$ and on $I_{4}, I_{5}$ were assumed to be independent and additive; (2) the dependence on $I_{1}, I_{2}, I_{3}$ was assumed to be the same as that of a slightly compressible, isotropic, neo-Hookean hyperelastic solid, with shear modulus $G$ and bulk modulus $K$. These restrictions lead to the following expression for the strain energy density

$$
W=\frac{1}{2} K(J-1)^{2}+\frac{1}{2} G(\operatorname{tr} \tilde{\boldsymbol{C}}-3)+W_{\mathrm{a}}\left(I_{4}, I_{5}\right)
$$

invoking the volume ratio $J \equiv \operatorname{det} \boldsymbol{F}=\sqrt{I_{3}}$ and the isochoric part of the right CauchyGreen tensor $\tilde{\boldsymbol{C}} \equiv \boldsymbol{J}^{-2 / 3} \boldsymbol{C}$. In equation (2), all anisotropy is captured by the third term $W_{\mathrm{a}}\left(I_{4}, I_{5}\right)$. We may note from equations (1) that $I_{4}=C_{33}=\lambda^{2}$, where $\lambda$ is the axial stretch of the tendon, while $I_{5}=C_{13}^{2}+C_{23}^{2}+C_{33}^{2}$, where subscripts $1,2,3$ refer to directions $X, Y, Z$.

To put this model in perspective, we note that several 3D hyperelastic constitutive models for tendons and ligaments of similar form to equation (2) have been proposed and discussed previously, from the pioneering work of Weiss et al. [23] onwards. Equation BIO-15-1191 Zavatsky 
(2) is essentially the same strain energy as that employed by some authors to model human knee ligaments (see [24, 25]), although these authors ignore the contribution of $I_{5}$. Note, however, that equation (2) differs in a small but important detail from that used by some other authors, who replaced $I_{4}$, or $I_{4}$ and $I_{5}$, by the corresponding invariants of $\tilde{C}$ (see $[23,26,27])$. That approach has the disadvantage that it can lead to un-physical predictions of material response $[28,29,30]$, and so it was not used here.

In transverse compression experiments such as those of the present study (where the sample is compressed over a length much greater than its thickness), axial constraint from friction between the tendon and the compression plates resists axial displacement, except close to the ends of the length of tendon under compression, where it emerges from the compression plates. Hence, throughout most of the tendon, all displacement is confined to the transverse plane and is independent of $Z$. Plane strain conditions then apply (i.e. $F_{i j}=0$ if $i$ or $j=3$ ) and $I_{4}=I_{5}=1$. Under these special circumstances, the third term in equation (2) makes no contribution to the strain energy of the specimen, and hence to the compressive load. The first two terms alone form the strain energy of an isotropic, slightly compressible neo-Hookean solid [20], which is therefore how tendon tissue was approximated in the present finite element simulations. Clearly this is an approximate description of the tendon, as the end-effect has been neglected. However, the resulting error is quantifiable and small, as shown in the Appendix (section A.2).

The glass plate and the glass backing were modelled as rigid surfaces. Contact between the tendon and these surfaces was modelled using a penalty method, invoking Coulomb friction with $\mu_{\mathrm{s}}=0.35$ (see above). In each simulation, a total $Y$-displacement of $0.92 \mathrm{~mm}$ (to exceed the maximum $Y$-displacement in the experiments) was applied to 
Journal of Biomechanical Engineering

the glass plate in 10 equal steps, placing the tendon in transverse compressive loading. The calculated reaction force on the glass plate was computed for each displacement.

For the idealized tendon model, an iterative sequence of simulations was carried out within a MATLAB optimization routine to find optimal values of the two parameters of the neo-Hookean model (Young's modulus $E=3 G /\left(1+\frac{1}{3} G / K\right)$ and Poisson's ratio $\left.v=0.5\left(1-\frac{2}{3} G / K\right) /\left(1+\frac{1}{3} G / K\right)\right)$, that minimized the r.m.s. difference between the averaged experimental, force versus $10 \mathrm{~s}$ isochronal creep displacement data, and the corresponding finite element simulation results ${ }^{4}$. Starting values of $E=0.4 \mathrm{MPa}$ and $v=$ 0.49 were chosen based on preliminary modelling investigations and values found in the literature [2]. The optimisation process took $c a 40$ minutes.

For the tendon-specific models, a similar process was followed, except that the iterative parameter-fitting was carried out for only $E$ in these cases, with $v$ held fixed at 0.49 , in order to reduce the computational burden. This was attempted for all tendons studied, but in many cases the difficulty of satisfying contact conditions simultaneously on the opposing irregularly-shaped surfaces of the tendon meant that convergence could not be achieved. Nevertheless, for two tendons full simulations and optimization were achieved. The process took $c a 8.3$ hours for each tendon.

\section{Results}

During each experimental loading profile, all tendons showed time-dependent creep and recovery in both $X$ - and $Y$-directions, in response to application and removal of

\footnotetext{
${ }^{4}$ The origin of displacement for experiments and simulations was taken as the displacement at a load of 0.9 N.

BIO-15-1191

Zavatsky
} 
Journal of Biomechanical Engineering

each load. Example data are shown in Fig. 3. All other tendons showed similar responses. In each case, the measured displacements in each test showed clear dependence on the load magnitude and on the time elapsed since its application or removal. In this paper, however, we focus only on the relation between measured displacements and load magnitude, for constant values of the creep time. Time-dependence of the displacements during creep and recovery will be discussed by the authors elsewhere.

Restricting attention to only isochronal creep displacements, the tendon was modelled as elastic - see Appendix (section A.1). Thus measurements of isochronal creep displacement, measured $10 \mathrm{~s}$ after application of each load, were used in determining the effective elastic constants for this creep time. The set of $10 \mathrm{~s}$ isochronal displacements in $Y$ - and $X$-directions for all seven tendons is shown in Fig. 4, plotted versus the applied transverse load. All tendon samples gave similar curves, but it is clear that magnitudes of the displacements varied significantly from tendon to tendon. Also visible in Fig. 4 is a trend to significant upwards curvature in the isochronal plots, indicating stiffening with increasing load.

The results of the inverse analyses, combining finite element simulations and experimental data to find best-fit values of elastic constants, were as follows. The idealized tendon model, combined with the averaged $10 \mathrm{~s}$ isochronal displacement data, gave $E=0.31 \pm 0.01 \mathrm{MPa}$ and $v=0.490 \pm 0.005$. The resulting calculated curves of force versus displacement (in $Y$ - and $X$-directions) are shown in Fig. 5, together with the measured force-displacement data. The two successful tendon-specific models, combined in each case with their corresponding $10 \mathrm{~s}$ isochronal displacement data, with $v=0.490$ imposed, gave $E=0.14 \pm 0.01 \mathrm{MPa}$ and $E=0.10 \pm 0.01 \mathrm{MPa}$. The resulting computed 
Journal of Biomechanical Engineering

curves are compared with the corresponding experimental data in Fig. 6. It is clear that the calculated curve in Fig. 6a approximates the experimental data to well within the reproducibility of displacement measurements quantified above (Section 2.2). However, Fig. $6 \mathrm{~b}$ shows greater deviation between the computed curve and the data: specifically the computed curve under-predicts the observed strain-stiffening.

\section{DISCUSSION}

\subsection{Methodology}

The present method of studying tendons in transverse compression has several advantages compared to the few previous attempts. It allows the testing of significant lengths of whole tendons, avoiding the need to cut out small test specimens with the attendant possibility of damage. It also enables the prior cross-sectional shape and size of the tendon to be determined in situ, after pre-conditioning and immediately before the start of testing. This is needed for realistic numerical simulations, which are essential in interpreting results from tests such as these, characterized by non-uniform stress. The method also provides displacement measurements in both transverse directions, parallel and perpendicular to the load, enabling two elastic constants to be determined from each data set. The present test rig is designed for dead-weight loading suited to creep tests, but could be readily adapted to displacement-controlled experiments.

As shown in Section 2.2, the experimental protocol produced excellent reproducibility of displacement $(40 \mu \mathrm{m})$ for repeat tests on a given pre-conditioned tendon sample. However, there was much greater variation than this between tendon samples, as seen in Fig. 4. This may reflect some difference in stiffness between tendons 
Journal of Biomechanical Engineering

from different animals and between tendons from the same animal, but there will certainly be a contribution from differences in cross-section size and shape between tendons after pre-conditioning - see Fig. 2.

In the FE simulations, the reader will recall an isotropic compressible neoHookean material was used as a model for the anisotropic tendons. The justification given in Section 2.3 was that the compressed portion of the tendon always had length:thickness $\sim 10: 1$. Hence axial friction between the tendon and the compression plates was expected to resist axial displacements, causing $I_{4}=I_{5}=1$ to be a good approximation within most of the tendon, in both experiments and simulations, justifying neglect of the $W_{\mathrm{a}}$ term in equation (2). Indeed, simulations were carried out with a range of values of $\mu_{\mathrm{s}}$. It was found that increasing $\mu_{\mathrm{s}}$ beyond the value 0.35 measured, and employed in the main simulations, produced negligible change in the predicted force for the range of displacements considered, demonstrating that frictional constraint was effectively complete for $\mu_{\mathrm{s}}=0.35$. Nevertheless, as shown in the Appendix, there were regions near the ends of the compressed segment of tendon where simulations with the isotropic constitutive model predicted unphysical levels of axial expansion. This endeffect is estimated to have caused a $c a+10 \%$ error in the apparent value of $E$ (see Appendix, section A.2): a significant systematic error, but small compared to the apparent scatter in $E$ between tendons.

\subsection{Isochronal displacement data}

The visible upward curvature of the experimental isochronal force-displacement plots bears a superficial resemblance to the well-known, and pronounced, strain- 
Journal of Biomechanical Engineering

stiffening seen at low strains in tensile tests on tendons and ligaments in the fiber-aligned direction. In that case, the primary cause of the nonlinearity is known: stiffening introduced at the microstructural level by straightening of initially crimped collagen fibres, and captured in the constitutive model of equation (2) through the anisotropic portion $W_{\mathrm{a}}$ of the strain energy function. In the case of plane-strain transverse compression, however, there is no such obvious microstructural explanation. But in fact none is needed. The model as proposed in equation (1), applied to plane strain transverse compression of a cylinder-like object such as a tendon, contains in-built geometrical sources of strain-stiffening.

The tendon cross-section changes shape during the non-uniform finite deformation. Specifically, the contact patch widens with increase in compressive load. For an example proof of this, see the analytical derivation for transverse compression of a linear elastic cylinder of circular cross-section given by Hadley et al. [8]. Widening of the contact patch produces strain-stiffening in a transversely compressed cylinder, even when the cylinder is linear elastic (see, for example, measurements and modeling of transverse compression of synthetic polymer fibers reported by Singletary et al. [14]). This adds to the stiffening effects of the finite reduction in thickness and increase in width of the tendon. In even a uniform plane strain compression test, the (linear) neo-Hookean model shows stiffening by approximately $10 \%$ over the strain-range of interest here.

Thus, it is no surprise that the present finite element model also demonstrates stiffening, without the addition of further terms in the constitutive model.

The differences between calculated and measured force-displacement nonlinearity can be attributed to variation in the fidelity with which the simulated tendons 
Journal of Biomechanical Engineering

captured details of the actual 3D tendon geometry, and hence the expansion of the contact patch under load. The analytical solution for transverse compression of a linear elastic circular cylinder $[8,10]$, predicts that the rate of growth of the width of the contact patch with respect to the compressive displacement, increases with decreasing radius of the contact. Therefore, stiffening arising from contact patch widening is expected to be sensitive to details of surface unevenness, possibly on too fine a scale to be captured by the 3D solid models used in the present FE modelling. A higher level of surface unevenness will give more pronounced stiffening. Another source of stiffening is axial expansion of the contact patch because of axial variation of the cross-section shape: this was captured relatively crudely in this work (only three cross-sections defined the segment of tendon under load). This probably explains why simulations with the idealised tendon model, even after optimization of elastic constants, tend to show less stiffening than the averaged experimental data from real, more irregularly shaped, tendons (compare Figs. 5 and 6). Such differences between tendon shapes also explain the pronounced differences in quality of fit between model predictions and experimental data, seen for the tendon-specific cases in Fig. 6. We may conclude that the tendon of Fig. 6a was well represented by its 3D solid model, while that of Fig. $6 \mathrm{~b}$ probably contained fine-scale surface unevenness across its width or along its length, not captured so well by its 3D solid model.

An additional, conceivable, source of variations in strain-stiffening between different tendons is that term 2 of the expression for $W$ in equation (2) is inaccurate, and in reality this term displays tendon-specific deviations from the neo-Hookean model. 
Such a possibility cannot be ruled out, but the present results can be adequately explained without it, and there seems to be no physical reason to invoke it.

Previous evidence on stress-strain non-linearity in transverse compression and tension testing of tendons and ligaments is contradictory. Quapp and Weiss [31] in tension tests on human MCL, and Williams et al. [3] in compression tests on rabbit patellar tendon, saw little evidence for non-linearity over the nominal strain range explored in the present work (0-0.15), but Bonafasi-Lista et al. [32] in tension tests on human MCL did see significant strain-stiffening. We conclude that, on the basis of the new data and other evidence available, the strain energy function in equation (2) is an adequate first approximation for modelling the transverse compression of tendons, but there remains an element of doubt in the case of transverse tension.

The values of optimized $E$ and $v$ found by comparing numerical simulations and experimental data have significance in two senses. Firstly, they represent the best-fit estimates of these two parameters of the isotropic part of the constitutive model in equation (2) obtainable from the data shown here - either with the aid of the idealized tendon model and averaged data, or with tendon-specific solid models and tendonspecific data. They will be useful as a guide for future numerical modelling of tendons using the model in equation (1). Secondly, they provide unambiguous evaluations of certain linear elastic constants of the tendon material itself (irrespective of the constitutive model used to describe it at finite strains). In the usual contracted notation for anisotropic linear elasticity (for example, see Nye [33]), taking subscripts 1,2,3 to refer to axes $X, Y, Z$, the fit of the idealized tendon model to the averaged data set gives the following terms of the 3D stiffness matrix of tendon: $c_{11}=c_{22}=\frac{4}{3} G+K=7.1 \pm 3.7 \mathrm{MPa}$; 
Journal of Biomechanical Engineering

$c_{12}=c_{21}=K-\frac{2}{3} G=6.9 \pm 3.7 \mathrm{MPa}$;

$c_{66}=G=0.104 \pm 0.004 \mathrm{MPa}$.

The only one of these for which there is a literature value for direct comparison is the transverse plane shear stiffness $c_{66}$, that was measured indirectly for human digital flexor tendons by Main et al. [7]. As mentioned in the Introduction, these authors' results imply $c_{66}=0.018 \mathrm{MPa}$ : a shear stiffness significantly lower than those obtained in the present work. The discrepancy may indicate a genuine difference between tendons from the two sources, or may reflect the difficulty of correctly interpreting data obtained using the test geometry of Main et al.

There are no other literature values of elastic constants able to be compared unambiguously with the present results. However, if $W_{\mathrm{a}}$ contributes negligible axial stiffness under axial contraction $(\lambda<1)$, as assumed in modelling by several previous authors and as possibly supported by experimental data for rabbit patella tendon [3], then measurements of transverse tensile Young's modulus $E_{\perp \mathrm{T}}$ are equivalent to the modulus $E$ determined in the present work. Lynch et al. [2] found the range $E_{\perp \mathrm{T}}=0.031-0.095$ $\mathrm{MPa}$, over the range of timescales employed in their work and at the smallest strains, in sheep flexor tendons, which (taking account of experimental uncertainty) is close to, but a little below the range of values for $E=0.10-0.31 \mathrm{MPa}$ found by the three routes in the present work. On the other hand, if $W_{\mathrm{a}}$ contributes an axial stiffness much larger than the other stiffnesses in axial extension $(\lambda>1)$, then measurements of transverse compression Young's modulus $E_{\perp \mathrm{C}}$ should equate to the plane strain transverse stiffness obtainable from the present results $=4 G\left(1+\frac{1}{3} G / K\right) /\left(1+\frac{4}{3} G / K\right)=0.13-0.41 \mathrm{MPa}$. Williams et 
Journal of Biomechanical Engineering

al. [3] obtained $E_{\perp \mathrm{C}}=0.014-0.020 \mathrm{MPa}$ for rabbit patella tendon, which lies considerably below the present results and those of Lynch et al. [2]. It possibly suggests genuine differences between tendons from different sources.

Given the similarities of their compositions, it might be expected that the transverse Young's moduli of ligaments are similar to those of tendons. However, the measurements so far reported for ligaments (all for human MCL, and all measured in transverse tension) range extremely widely, with values of $E_{\perp \mathrm{T}}$ varying from $0.01-0.03$ $\mathrm{MPa}$ (as deduced from oscillatory tests superposed on a $4 \%$ static strain by Bonifasi-Lista et al. [32]) to $11 \mathrm{MPa}$ (as deduced from constant strain-rate tests at $0.017 \mathrm{~s}^{-1}$ by Quapp and Weiss [31]). Thus, although they do span the range of values of $E$ found in the present work, no useful conclusions can yet be drawn on how they compare to results for tendon.

\section{CONCLUSIONS}

The work described here has demonstrated a new method for studying the compression of tendons transverse to their fiber-aligned axes. A length of tendon much greater than its width is compressed between two parallel, rigid, flat plates. The method follows a procedure similar to that used previously with success for synthetic polymer fibers. It offers several advantages compared to methods previously applied to tendons. When it is applied to tendons, however, deformations can be relatively large and crosssections are usually somewhat irregular. Therefore quantitative evaluations of elastic constants require the use of inverse analysis, employing numerical simulation of tests and the fitting of simulations to measurements. Moreover, such simulations must be supplied 
Journal of Biomechanical Engineering

with the 3D geometry of the tendon. The present method allows the geometry to be determined as an integral step within the test procedure, by employing image-analysis as proposed previously by the authors. This approach allows the geometry to be measured in situ, after pre-conditioning and immediately prior to testing, as required.

In the finite element simulations of the present study, a hyperelastic constitutive model was invoked, with a two-part strain energy density function: a slightly compressible isotropic neo-Hookean part, and an anisotropic part $W_{\mathrm{a}}$, dependent only on $C_{i 3}(i=1 \ldots 3)$. The advantage of this approach when applied to the present experimental method is that no knowledge of $W_{\mathrm{a}}$ is needed since, as a first approximation, these terms in $\boldsymbol{C}$ do not change during testing.

Results of the inverse analysis, in which finite element predictions of load versus displacement were fitted to measurements of load versus isochronal creep displacement, were Young's modulus and Poisson's ratio for the neo-Hookean part of the constitutive response. For the bovine digital extensor tendons of the present work, these are respectively: $E=0.31 \mathrm{MPa}, v=0.49$, as found using averaged $10 \mathrm{~s}$ isochronal displacement data, and an idealized, representative 3D geometry for the tendon: a cylinder with race-track shaped cross-section, fitted to the averaged measured crosssection shape. From these values, representative values of specific terms in the stiffness matrix of the present tendons can be deduced (in terms of contracted notation, with axes $1,2,3$ corresponding to axes $X, Y, Z): \quad c_{11}=c_{22}=7.1 \mathrm{MPa}, \quad c_{12}=c_{21}=6.9 \mathrm{MPa}$, $c_{66}=0.10 \mathrm{MPa}$. Data for two specific tendons, using their actual measured cross-sections and assuming $v=0.49$, gave the values $E=0.14$ and $0.10 \mathrm{MPa}$. 
Journal of Biomechanical Engineering

Some assumptions and approximations were made in their determination (see above, and the Appendices), hence all these numerical values are subject to some uncertainty additional to that arising from experimental scatter. Nevertheless, some trends are clear. The moduli obtained here for bovine digital extensor tendons exceed values reported previously for other tendons, by factors ranging from $\mathrm{ca} 3$ to $\mathrm{ca} 16$, but lie within the extremely wide range of values (varying by more than a factor of 100) previously found for ligaments. The reasons for all these considerable variations are not known. Some may be true reflections of property variations between tendons of different origin, but it is likely that some arise from difficulties in execution and accurate interpretation of the challenging experiments previously used in their determination.

Isochronal force-displacement curves, as measured and as simulated, showed varying degrees of stiffening with increasing load. In terms of the analysis used here employing the neo-Hookean material model, the causes of stiffening are geometrical. The tendon's cross-sectional shape and contact patch change under load, depending on details of the initial 3D tendon shape, and there is the usual finite reduction of thickness and increase in width associated with finite plane strain compression.

\section{APPENDIX: Approximations invoked}

Several aspects of the 3D mechanical response of tendons remain unknown. In the present work therefore, to isolate particular features of interest in the response to transverse compression, it was necessary to invoke two significant approximations. These are re-stated below, together with comments and justifications. 
Journal of Biomechanical Engineering

\section{A.1 The elastic approximation}

Time-dependence associated with the full 3D nonlinear viscoelasticity of tendons is not yet known. Therefore it could not be included within the constitutive model employed in the inverse analysis used here. Instead, in finite element simulations, the $10 \mathrm{~s}$ isochronal creep response of the tendon was modelled as being the same as an elastic response. An elastic constitutive model was employed; and the predicted load versus displacement relations were fitted to experimental measurements of creep load versus 10s isochronal displacement. The following provides some justification for this approach.

If the current constitutive model were extended to include viscoelasticity, the Cauchy stress response would still consist of an isotropic contribution and an anisotropic contribution. Under plane strain conditions (approximating the present tests, see below) only the isotropic response would contribute to the applied load. Let us invoke three simplifying assumptions. (1) Linear viscoelasticity applies: compression of the tendon corresponds to plane strain compression of an isotropic linear viscoelastic solid. (2) The isotropic contribution to the viscoelastic response is synchronous: shear and hydrostatic creep compliances in the reference configuration have the same time dependence (viz. their ratio is independent of time). (3) The width of the contact patch is constant under load. The elastic-viscoelastic Correspondence Principle [34] then guarantees that the stress distribution within the tendon at any given time is identical to that applying if the tendon were linear elastic, for the applied load acting at that time. For step-wise loading as used in creep tests, all strains, and hence $X$ and $Y$-direction displacements, at a given creep time are equal to those in a linear elastic material with the same Poisson's ratio and a Young's modulus equal to the reciprocal isochronal tensile creep compliance, for the 
Journal of Biomechanical Engineering

corresponding creep time. This may be shown, for example, by applying the Correspondence Principle to the analytical solution for transverse compression of an elastic cylinder - see the work of Ward and co-workers [8]-[10].

It is clear that the assumptions made here do not reflect reality exactly. The present experiments extend beyond the linear viscoelastic region of strain, and some time-dependence of Poisson's ratio may be expected. In addition, during creep the contact patch width gradually increases. An estimate of this may be quantified by applying the Correspondence Principle to the analytical solution for the contact patch width of an elastic cylinder - see Hadley and Ward [8]. The time dependence of the tendons' creep response has been analyzed by the authors and will be discussed elsewhere (to be published). From this information it may be shown that, for the racetrack geometry of Figure 2(b), the contact patch will grow by less than $3 \%$ between $1 \mathrm{~s}$ and $100 \mathrm{~s}$ during the creep tests, so this error is small. Therefore equivalence between the elastic case modelled and the isochronal viscoelastic measurements is not exact, but is a necessary and reasonable approximation.

\section{A.2 The plane strain approximation}

The present experiments were designed to minimize the anisotropic contribution to the stress (term 3 in equation (2)), by imposing approximately plane strain conditions: compression of a length of tendon much greater than its thickness. Friction on the compression plates then resisted axial expansion, in the experiments and in simulations. In addition, in the experiments plane strain was assured by the intrinsic anisotropy of the tendon. If $v_{23}$ is the Poisson's ratio governing axial expansion under a transverse 
compression and $E_{3}$ is the small strain (toe region) Young's modulus in the fiber-aligned direction, thermodynamic constraint on the elastic constants requires [35] $v_{23}<\sqrt{E / E_{3}}$. Tendons from the same batch as those of the present study were tested in axial tension [19] and gave a mean over nine tendons of $E_{3}=360 \mathrm{MPa}$. Therefore the values of $E=$ 0.1-0.3 MPa obtained in the present work indicate $v_{23}<0.01$. It is clear, therefore, that tendons, even in the absence of friction, do not expand axially to a significant degree when compressed transverse to their axes.

By contrast, in the present FE simulations an isotropic constitutive model was employed and friction provided the only restriction on axial expansion. This gave plane strain conditions near the center of the $40 \mathrm{~mm}$ wide compression zone, but simulated tendons expanded axially near the extremities of this zone. Such an unphysical end-effect inevitably introduces an error when comparing simulations with the responses of real tendons which did not expand axially. We may estimate the magnitude of the error from the simulated distribution of axial strain. Fig. 7 shows an example contour plot of the calculated axial true strain $\varepsilon_{z}(=\ln \lambda)$ in the mid-section $Y-Z$ plane. Let $\lambda_{x}$ and $\lambda_{y}$ represent the local $X$ and $Y$-direction transverse stretches $\left(\lambda_{y}<1\right)$. Then, neglecting compressibility of the tendon, we have $\lambda_{x}=\left(\lambda \lambda_{y}\right)^{-1}$ and in addition $\lambda_{y}$ is constrained by the known $\Delta Y=\int_{0}^{\Delta Y_{0}} \lambda_{y} \mathrm{~d} y$. If we also neglect here any $X$-wise stress, this provides enough information to use the neo-Hookean constitutive model to calculate the local $Y$ direction transverse stress and hence the simulated transverse load on the compression plates (which can then be checked by comparison with the FE calculation). For the case 
Journal of Biomechanical Engineering

depicted in Fig. 7, the estimated load $F=40.5$ N. Repeating the calculation for a tendon of identical geometry and the same value of $E$, but imposing complete axial constraint $\lambda=1$ everwhere, gives $F=44.4 \mathrm{~N}$. This simple check reveals that incomplete axial constraint in the simulations caused a ca $10 \%$ under-prediction of applied load, and hence a $c a 10 \%$ over-estimation of the Young's modulus $E$ required to fit experimental data. This error is significant, but much smaller than the natural variation in value of $E$ between different tendons from the same source. 
Journal of Biomechanical Engineering

\section{References}

[1] Cook, J.L., and Purdam, C., 2013. "Is Compressive Load a Factor in the Development of Tendinopathy?," Br. J. Sports Med. 46, pp. 163-168.

[2] Lynch, H.A., Johannessen, W., Wu, J.P., Jawa, A., and Elliott, D.M., 2003. "Effect of Fiber Orientation and Strain Rate on the Nonlinear Uniaxial Tensile Material Properties of Tendon,” ASME J. Biomech. Eng. 125, pp. 726-731.

[3] Williams, L.N., Elder, S.H., Bouvard, J.L., and Horstemeyer, M.E., 2008. "The Anisotropic Compressive Mechanical Properties of the Rabbit Patellar Tendon," Biorheology 45, pp. 577-586.

[4] Lee, S-B., Nakajima, T., Luo, Z-P., Zobitz, M.E., Chang, Y-W., and An, K-N., 2000. "The Bursal and Articular Sides of the Suprinatus Tendon have Different Compressive Stiffness,” Clin. Biomech. 15, pp. 241-247.

[5] Ikeda, J., Zhao, C., Chen, Q., Thoreson, A.R., An, K-N., and Amadio, P.C., 2011. “Compressive Properties of cd-HA-gelatin Modified Intrasynovial Tendon Allograft in Canine Model In Vivo,” J. Biomech. 44, pp. 1793-1796.

[6] Dourte, L.M., Pathmanathan, L., Jawad, A.F., Iozzo, R.V., Mienaltowski, M.J., Birk, D.E., and Soslowsky, L.J., 2012. "Influence of Decorin on the Mechanical, Compositional, and Structural Properties of the Mouse Patellar Tendon," ASME J. Biomech. Eng. 134, pp. 031005-1 - 013005-8.

[7] Main, E.K., Goetz, J.E., Rudert, M.J., Goreham-Voss, C.M., and Brown, T.D., 2011. “Apparent Transverse Compressive Material Properties of the Digital Flexor Tendons and the Median Nerve in the Carpal Tunnel,” J. Biomech. 44, pp. 863-868. 
Journal of Biomechanical Engineering

[8] Hadley, D.W., Ward, I.M., and Ward, J., 1965. “The Transverse Compression of Anisotropic Fibre Monofilaments,” Proc. Royal Soc. A 285, pp. 275-286.

[9] Pinnock, P.R., Ward, I.M., and Wolfe, J.M., 1966. "The Compression of Anisotropic Monofilaments,” Proc. Royal Soc. A 291, pp. 267-278.

[10] Abdul Jawad, S., and Ward, I.M., 1978. "Transverse Compression of Oriented Nylon and Polyethylene Extrudates,” J. Matls. Sci. 13, pp. 1381-1387.

[11] Chakravarty, A.C., 1969. "Observations on Transverse Compression of Some Plant Fibers,” Textile Res. J. 39, pp. 878-881.

[12] Morris, S., 1968. "The Determination of the Lateral Compression Modulus of Fibres,” J. Textile Inst. 59, pp. 536-547.

[13] Phoenix, S.L., and Skelton, J., 1974. "Transverse Compression Moduli and Yield Behavior of Some Orthotropic, High-Modulus Filaments,” Textile Res. J. 44, pp. 934-940.

[14] Singletary, J., Davis, H., Ramasubramanian, M.K., Knoff, W., and Toney, M., 2000. “The Transverse Compression of PPTA Fibers,” J. Matls. Sci. 35, pp. 573-581.

[15] Salisbury, S.T.S., Buckley, C.P., and Zavatsky, A.B., 2008. "Image-Based NonContact Method to Measure Cross-Sectional Areas and Shapes of Tendons and Ligaments,” Meas. Sci. Tech. 19, 045705 (9pp).

[16] Woo, S.L-Y., Orlando, C.A., Camp, J.F., and Akeson, W.H., 1986. "Effects of Post-Mortem Storage by Freezing on Ligament Tensile Behavior,” J. Biomech. 19, pp. 399-404. 
Journal of Biomechanical Engineering

[17] Chimich, D., Shrive, N., Frank, C., Marchuk, L., and Bray, R., 1992. "Water Content Alters Viscoelastic Behaviour of the Normal Adolescent Rabbit Medial Collateral Ligament,” J. Biomech. 25, pp. 831-837.

[18] Hayward, A.T.J., 1977. Repeatability \& Accuracy. Mechanical Engineering Publications, London.

[19] Salisbury, S.T.S., 2008. The Mechanical Properties of Tendon. D.Phil. thesis, University of Oxford.

[20] Abaqus, 2005. Abaqus Theory Manual, vol. 6.5-1, chapter 3.1.1, ABAQUS Inc., RI, USA.

[21] Spencer, A.J.M., 1972. Deformations of Fibre-Reinforced Materials. Oxford University Press, London.

[22] Holzapfel, G.A., 2000. Nonlinear Solid Mechanics: A Continuum Approach for Engineering. John Wiley \& Sons, Chichester, UK.

[23] Weiss, J.A., Maker, B.N., and Govindjee, S., 1996. "Finite Element Implementation of Incompressible, Transversely Isotropic Hyperelasticity," Comp. Meth. Appl. Mech. Eng. 135(1-2), pp. 107-128.

[24] Peña, E., Calvo, B., Martinez, M.A., and Doblare, M., 2006. “Three Dimensional Finite Element Analysis of the Combined Behaviour of Ligaments and Menisci in the Healthy Human Knee Joint,” J. Biomech. 39, pp. 1686-1701.

[25] Mohamed, A-N.A., Brown, M.A., and Shabana, A.A., 2010. "Study of the Ligament Tension and Cross-Section Deformation Using Nonlinear Finite Element/Multibody System Algorithms,” Multibody Sys. Dyn. 23, pp. 227-248. 
Journal of Biomechanical Engineering

[26] Gardiner, J.C., and Weiss, J.A., 2003. "Tendon-Specific Finite Element Analysis of the Human Medial Collateral Ligament During Valgus Knee Loading,” J. Orthop. Res. 21, pp. 1098-1106.

[27] Limbert, G., Middleton, J., and Taylor, M., 2004. "Finite Element Analysis of the Human ACL Subjected to Passive Anterior Tibial Loads," Comp. Methods Biomech. Biomed. Eng. 7, pp. 1-8.

[28] Sansour, C., 2008. "On the Physical Assumptions Underlying the VolumetricIsochoric Split and the Case of Anisotropy,” Europ.J. Mech. A/Solids 27, pp. 28-39.

[29] Helfenstein, J., Jabareen, M., Mazza, E., and Govindjee, S., 2010. "On NonPhysical Response in Models for Fiber-Reinforced Hyperelastic Materials," Int. J. Solids Struct. 47, pp. 2056-2061.

[30] Gilchrist, M.D., Murphy, J.G., Parnell, W., and Pierrat, B., 2014. "Modelling the slight compressibility of anisotropic soft tissue,” Int. J. Solids Struct. 51, pp. 38573865 .

[31] Quapp, K.M., and Weiss, J.A., 1998. "Material Characterization of Human Medial Collateral Ligament,” ASME J. Biomech. Eng. 120, pp. 757-763.

[32] Bonifasi-Lista, C., Lake, S.P., Small, M.S., and Weiss, J.A., 2005. "Viscoelastic Properties of the Human Medial Collateral Ligament Under Longitudinal, Transverse and Shear Loading,” J. Orthop. Res. 23, pp. 67-76.

[33] Nye, J.F., 1985. Physical Properties of Crystals. Oxford University Press, Oxford.

[34] Lakes, R., 2009. Viscoelastic Materials. Cambridge University Press, New York.

[35] Cowin, S.C., and Van Buskirk, W.C., 1986. "Thermodynamic restrictions on the elastic constants of bone," J. Biomech. 19, pp. 85-87. 
Journal of Biomechanical Engineering

\section{ACKNOWLEDGMENT}

This work was supported in part by the Leverhulme Trust (UK). 


\section{Figure Captions}

Fig. 1 Schematic drawing of the tendon compression rig. (a) View in $X-Y$ plane. Distance from paddle pivot to center of the glass window $=175 \mathrm{~mm}$, distance from center of glass window to LVDT $=125 \mathrm{~mm}$, distance from LVDT to load $=50 \mathrm{~mm}$. (b) View in $Y-Z$ plane. Height of glass plate $=40$ $\mathrm{mm}$, height of glass backing $=75 \mathrm{~mm}$.

Fig. 2 (a) Tendon cross-sectional profiles acquired after preconditioning. Tendon width $\left(\Delta X_{0}\right)$ ranged from 8.9 to $15.6 \mathrm{~mm}$, and tendon thickness $\left(\Delta Y_{0}\right)$ ranged from 3.7 to $5.8 \mathrm{~mm}$ (correctly resolved to within $0.15 \mathrm{~mm}$ or less [15]). (b) Race-track approximation (cross-sectional area $36.6 \mathrm{~mm}^{2}$ ) fitted to the average tendon shape (cross-sectional area $35.1 \mathrm{~mm}^{2}$ ).

Fig. 3 Examples of measured displacements in (a) $Y$-direction and (b) $X$ direction as functions of time, during each profile of transverse loading. Each curve begins at time zero and is followed by a brief spike, which indicates when the $0.9 \mathrm{~N}$ calibration load was applied; the displacement axis is zeroed at the peak of this spike. Following the spike, there is a brief period of recovery followed by the main load applied at $90 \mathrm{~s}$.

Fig. $4 \quad$ Load versus $10 \mathrm{~s}$ isochronal (a) $Y$-displacement and (b) $X$-displacement measured during creep for seven tendons.

Fig. 5 Comparison of the finite element simulation of transverse compression of a model tendon with race-track geometry (see Fig. 2) (solid lines), with average measured $10 \mathrm{~s}$ creep displacements (symbols) in (a) the $Y$ - 
Journal of Biomechanical Engineering

direction and (b) the $X$-direction. Dashed lines show the range of measured displacements at each load level. The model uses best fit values $E=0.34 \mathrm{MPa}$ and $v=0.490$

Fig. 6 Comparison of the finite element simulations (solid lines) of transverse compression of two tendons, using their tendon-specific cross-sectional geometries, with measured $10 \mathrm{~s}$ creep displacements (symbols) in the $Y$ direction. The model uses $v=0.49$, and best fit values (a) $E=0.14 \mathrm{MPa}$ and (b) $E=0.10 \mathrm{MPa}$.

Fig. 7 Example of a finite element model of the experiment viewed from the side ( $Y-Z$ plane, as defined in Fig. 1). The tendon is being compressed between the glass plate and the glass backing by $19 \%$. Logarithmic (true) tensile strain in the fiber-aligned direction $\left(\varepsilon_{Z}\right)$ is plotted, revealing some axial expansion at the ends of the compression zone. 
Journal of Biomechanical Engineering

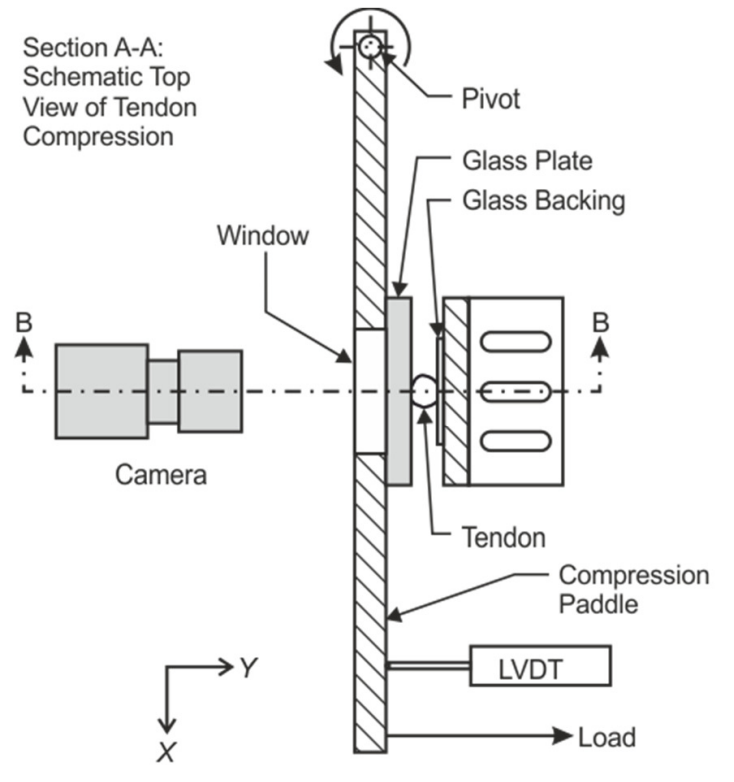

(a)

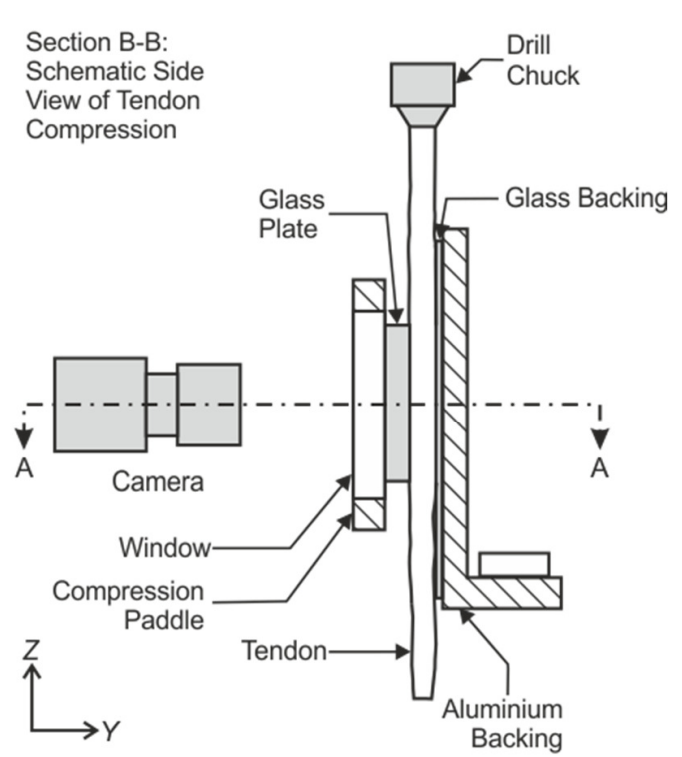

(b)

Figure 1 
Journal of Biomechanical Engineering

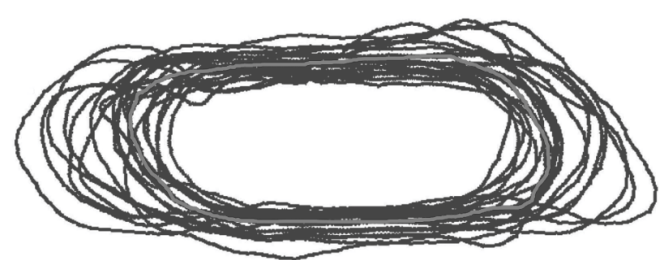

(a)

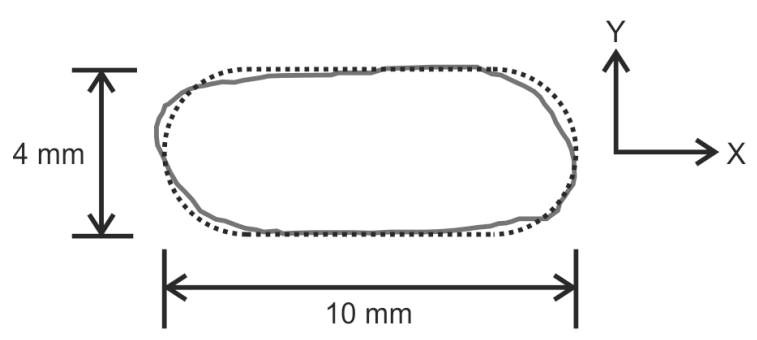

(b)

Figure 2 
Journal of Biomechanical Engineering
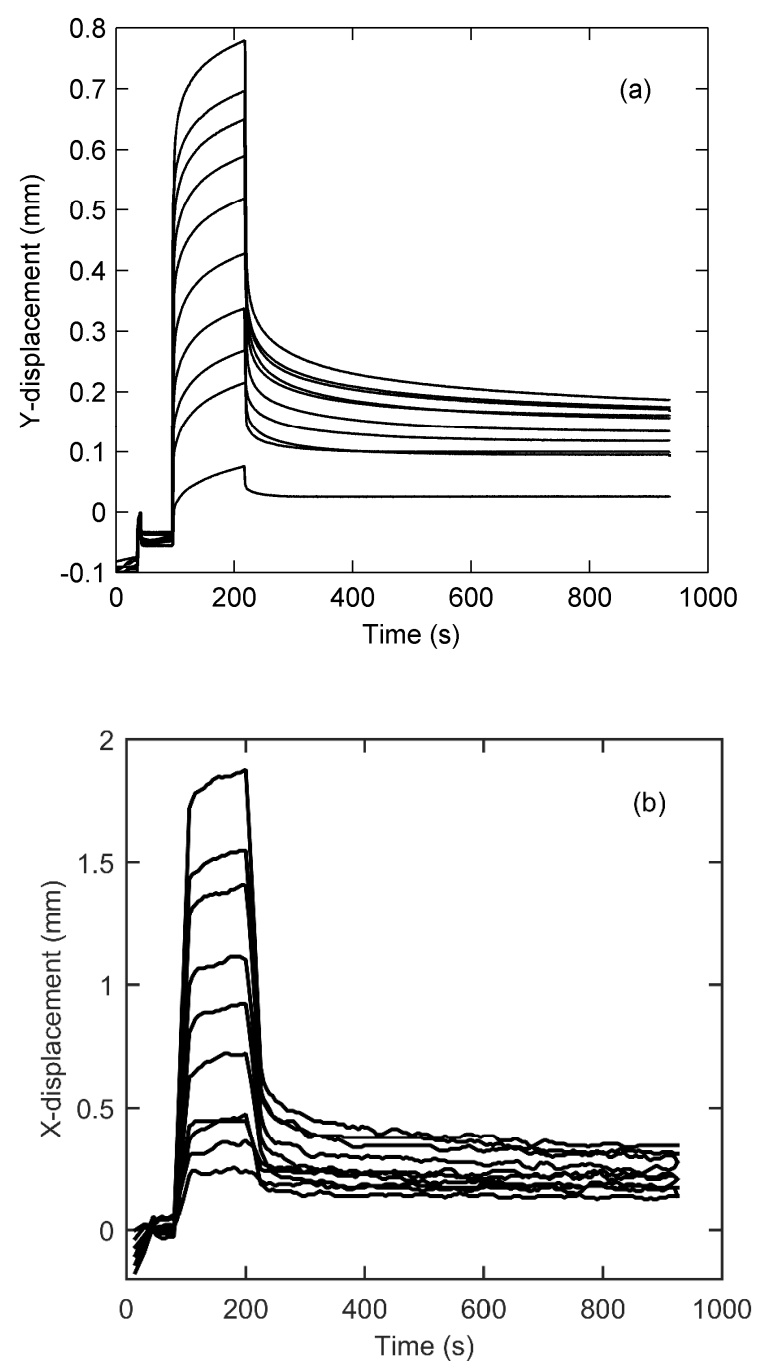

Figure 3 
Journal of Biomechanical Engineering
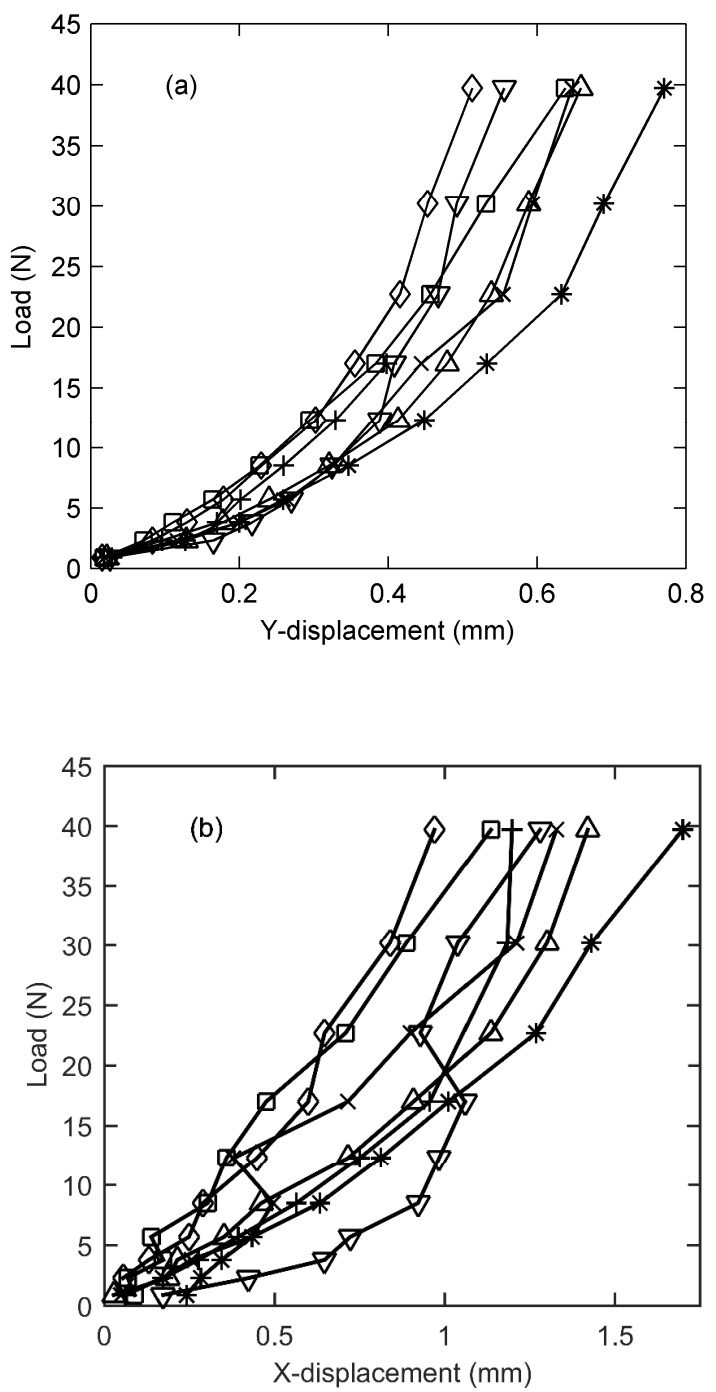

Figure 4 
Journal of Biomechanical Engineering
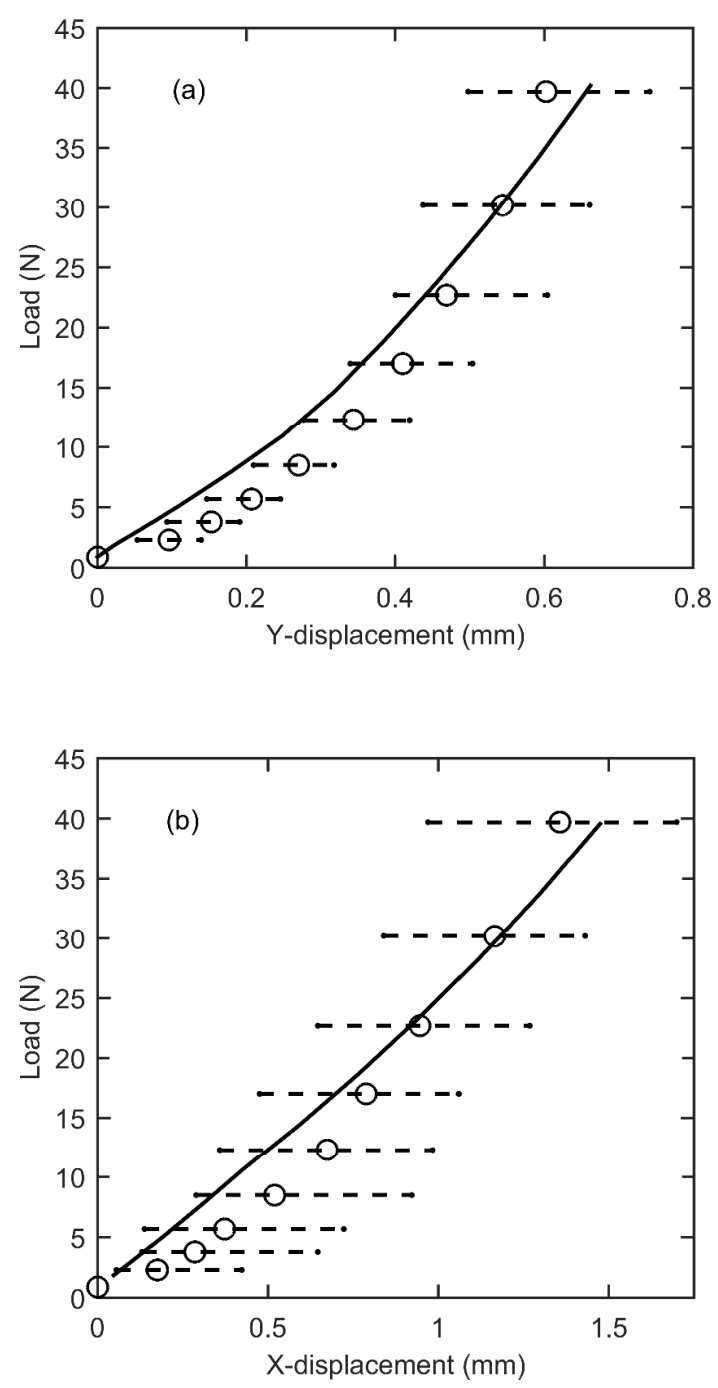

Figure 5 
Journal of Biomechanical Engineering
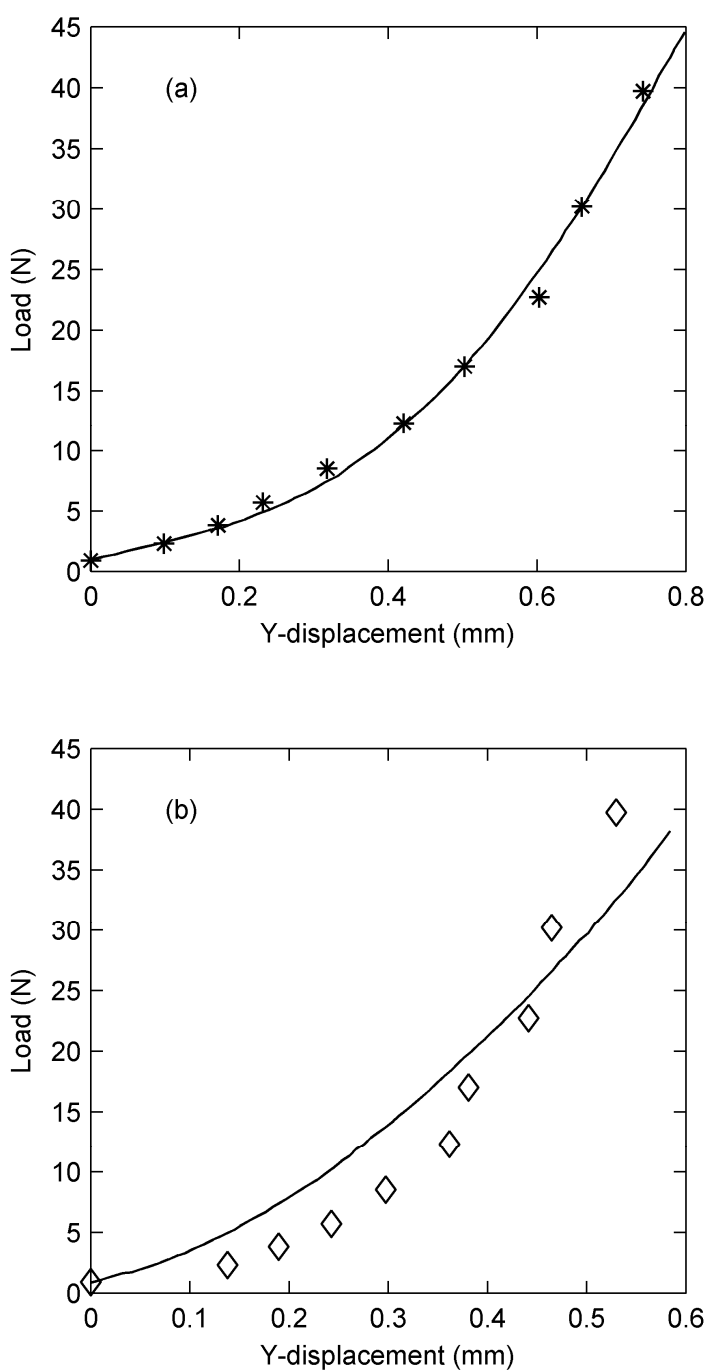

Figure 6 
Journal of Biomechanical Engineering

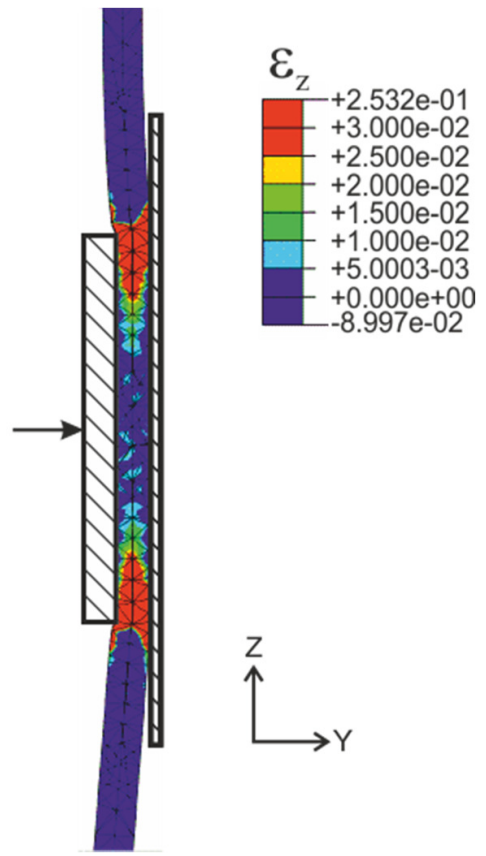

Figure 7

BIO-15-1191 